University of South Carolina

Scholar Commons

\title{
Study of Ionic Conductivity Profiles of the Air Cathode of a PEMFC by AC Impedance Spectroscopy
}

\author{
Qingzhi Guo \\ University of South Carolina - Columbia \\ Maria Cayetana \\ Yu-Min Tsou \\ Emory S. De Castro \\ Ralph E. White \\ University of South Carolina - Columbia, white@cec.sc.edu
}

Follow this and additional works at: https://scholarcommons.sc.edu/eche_facpub

Part of the Membrane Science Commons

\section{Publication Info}

Published in Journal of the Electrochemical Society, Volume 150, Issue 11, 2003, pages A1440-A1449. (c) The Electrochemical Society, Inc. 2003. All rights reserved. Except as provided under U.S. copyright law, this work may not be reproduced, resold, distributed, or modified without the express permission of The Electrochemical Society (ECS). The archival version of this work was published in Guo, Q., Cayetana, M., Tsou, Y., De Castro, E.S., \& White, R.E. (2003). Study of Ionic Conductivity Profiles of the Air Cathode of a PEMFC by AC Impedance Spectroscopy. Journal of the Electrochemical Society, 150(11): A1440- A1449.

Publisher's Version: http://dx.doi.org/10.1149/1.1612502

This Article is brought to you by the Chemical Engineering, Department of at Scholar Commons. It has been accepted for inclusion in Faculty Publications by an authorized administrator of Scholar Commons. For more information, please contact digres@mailbox.sc.edu. 


\title{
Study of Ionic Conductivity Profiles of the Air Cathode of a PEMFC by AC Impedance Spectroscopy
}

\author{
Qingzhi Guo, ${ }^{\mathrm{a}, *}$ Maria Cayetano, ${ }^{\mathrm{b}}$ Yu-min Tsou, ${ }^{\mathrm{b}, * *}$ Emory S. De Castro, ${ }^{\mathrm{b}, * *}$ \\ and Ralph E. White $\mathrm{a}^{\mathrm{a}, * * *, \mathrm{z}}$
}

${ }^{a}$ Center for Electrochemical Engineering, Department of Chemical Engineering,

University of South Carolina, Columbia, South Carolina 29208, USA

${ }^{b}$ De Nora North America, Incorporated, E-TEK Division, Somerset, New Jersey 08873, USA

\begin{abstract}
A characterization of the ionic conduction of the active layer of a polymer electrolyte membrane fuel cell (PEMFC) cathode by ac impedance measurement at open-circuit potential conditions was conducted. Porous electrode theory was used to derive a compact equation, $\partial^{2} \hat{\Phi}_{2} / \partial y^{2}+\partial \ln f(y) / \partial y \times \partial \hat{\Phi}_{2} / \partial y-R / f(y)(1+j \Omega) \hat{\Phi}_{2}=0$, to solve for the impedance response of a cathode at open-circuit potential conditions. This equation includes a parameter $R$, the ratio of an ionic resistance (evaluated at the active layer/membrane interface), to the total charge-transfer resistance of the active layer. The influence of an assumed ionic conductivity distribution profile $f(y)$ on the error in the estimation of total double-layer capacitance of the active layer from the $-1 /\left(Z_{\mathrm{Im}} \omega\right)$ vs. $Z_{\mathrm{Re}}$ plot was also investigated in this work. The increase of ionic conductivity in the active layer of an air cathode with an increase in the ionomer loading was revealed from both impedance data and surface area measurements. A nonlinear parameter estimation method was used to extract the ionic resistance from the high-frequency region of the impedance data at open-circuit potential conditions. The assumed ionic conductivity distribution profile in the active layer was found to vary with ionomer loadings.
\end{abstract}

(C) 2003 The Electrochemical Society. [DOI: 10.1149/1.1612502] All rights reserved.

Manuscript submitted January 13, 2003; revised manuscript received May 12, 2003. Available electronically September 17, 2003.

The transport ability of protons to catalyst sites is greatly enhanced by impregnating a proton-conducting ionomer in the catalyst active layer of the cathode of a polymer electrolyte membrane fuel cell (PEMFC). ${ }^{1}$ The function of a proton-conducting ionomer such as Nafion ${ }^{\circledR}$ is to provide an ionic path for proton migration from the membrane/active layer interface to the catalyst sites.

Recently, Lefebvre et al. ${ }^{2}$ investigated the ionic conductivity profile inside the active layer of a cathode for a PEMFC by ac impedance spectroscopy. In their work, they first operated a cell at $0.5 \mathrm{~V}$ until a steady-state current was obtained. Then, they fed nitrogen to the cathode to replace $\mathrm{O}_{2}$ and measured the impedance response at $0.95 \mathrm{~V} v s$. the $\mathrm{H}_{2}$ anode after current interruption. A transmission line equivalent circuit model was used to treat the porous active layer of the cathode. The capacitance $v s$. resistance plot in their work showed that the curve first rose linearly with the increase of the real component of impedance response, and then went up nonlinearly before stopping at one fixed point. At the stopping point, the capacitance value was related to the total double-layer capacitance of the active layer. They also observed that the higher the ionomer content in the active layer, the steeper the slope of the linear part of the curve.

In their work, they considered a cathode with nonpolarizable nature; the gas fed to the cathode was replaced by nitrogen after a steady-state operation, and double-layer charging was the only way for charge transfer across the catalyst/electrolyte interface. A case where the electrochemical charge-transfer reaction is occurring together with the double-layer charging, which is true for a cathode fed with air or $\mathrm{O}_{2}$, was not considered in their work. In the present work, we seek to extend their work to include the charge-transfer reaction. We carried out impedance measurement on a system where air was fed to the cathode and developed an impedance model for this condition, where both the charge-transfer reaction and doublelayer charging are important. Since the transmission line equivalent circuit model is inconvenient to treat some parameters, such as the ionic conductivity considered in this work which value changes with spatial coordinate, we decided to use Newman's macroscopic porous electrode model $^{3}$ for the present system. This model treats the po-

\footnotetext{
* Electrochemical Society Student Member.

** Electrochemical Society Active Member

*** Electrochemical Society Fellow.

z E-mail: white@engr.sc.edu
}

rous electrode as the superposition of two continua, one represents the matrix phase and another represents the electrolyte phase. Even if a third phase, a gas phase, might also exist in the active layer for at least some air cathodes, we elected not to include this phase in our present model based on the assumption that there is a uniform concentration of $\mathrm{O}_{2}$ in the gas phase of the active layer. This assumption is very likely to hold for the impedance response at open-circuit potential (OCP) conditions. For simplicity, a one-dimensional model is considered here. This treatment is justified for the impedance study as supported by the work of Springer et al. ${ }^{4}$

Cyclic voltammetry (CV) was used in the literature ${ }^{5-7}$ for the in situ measurement of the active surface area of a gas diffusion electrode (GDE) by measuring the hydrogen adsorption and desorption charge on the Pt surface. The measured surface area from CV tests is correlated with the capacitance results found from the impedance data.

\section{Model Development}

The ionic conduction in the electrolyte phase of the active layer of an air cathode (see Fig. 1) is given by ${ }^{3,8}$

$$
i_{2}=-\kappa \frac{\partial \Phi_{2}}{\partial x}
$$

where $i_{2}$ is the ionic current density, $\kappa$ is the effective ionic conductivity, $\Phi_{2}$ is the electrolyte phase potential measured with a hypothetical $\mathrm{O}_{2}$ reference electrode placed locally, and $x$ is the spatial coordinate. The gradient of a concentration cell potential, which is related to the partial pressure variation of $\mathrm{O}_{2}$ in the gas pores, multiplied by $\kappa$ should also be included in Eq. 1, according to Newman's convention to define the electrolyte phase potential, since the electrolyte potential difference between two arbitrary points measured by using two $\mathrm{O}_{2}$ reference electrodes should be able to reflect the potential of a concentration cell. ${ }^{3}$ This gradient term is neglected here based on the assumption that this partial pressure is most likely to be uniform under OCP conditions.

Conservation of charge in the active layer is given $b^{3}$

$$
\frac{\partial i_{2}}{\partial x}=a\left(4 F j_{\mathrm{n}}+C_{\mathrm{dl}} \frac{\partial\left(\Phi_{1}-\Phi_{2}\right)}{\partial t}\right)
$$

where $C_{\mathrm{dl}}$ is the double-layer capacitance of the active layer per unit surface area of the catalyst Pt including not only a contribution from 


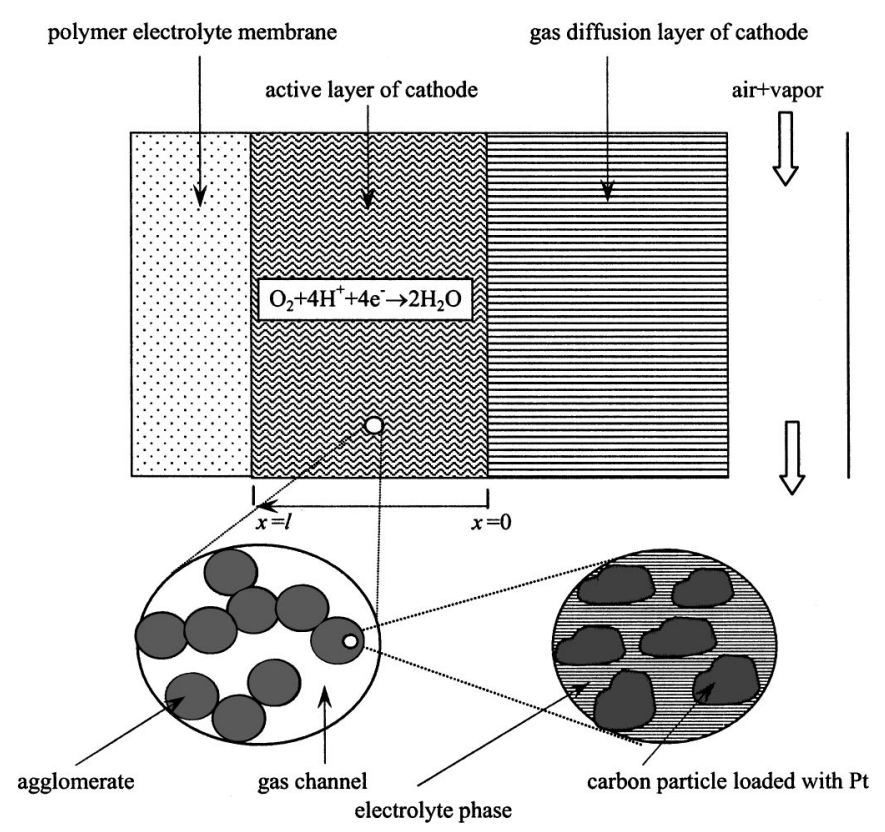

Figure 1. Schematic illustration of a cathode for the polymer electrolyte membrane fuel cell.

Pt but also a contribution from its carbon support, $\Phi_{1}$ is the matrix phase potential, $a$ is the active surface area of Pt per unit volume of the active layer, $j_{\mathrm{n}}$ is the wall flux of $\mathrm{O}_{2}$ on the catalyst Pt surface (the carbon surface is assumed here to be inert to $\mathrm{O}_{2}$ reduction), $4 \mathrm{~F}$ is the charge required for the reduction of one mole of $\mathrm{O}_{2}$ following a simple overall electrochemical reaction such as Eq. 3

$$
\mathrm{O}_{2}+4 \mathrm{H}^{+}+4 \mathrm{e} \rightarrow 2 \mathrm{H}_{2} \mathrm{O}
$$

and $4 F j_{\mathrm{n}}$ is assumed to be related to a general Butler-Volmer equation ${ }^{3,9}$ to describe a complicated kinetics under OCP conditions

$$
4 F j_{\mathrm{n}}=i_{0}\left\{\exp \left[\frac{\alpha_{\mathrm{a}} n F\left(\Phi_{1}-\Phi_{2}\right)}{R T}\right]-\exp \left[-\frac{\alpha_{\mathrm{c}} n F\left(\Phi_{1}-\Phi_{2}\right)}{R T}\right]\right\}
$$

where $\alpha_{\mathrm{a}}$ and $\alpha_{\mathrm{c}}$ are the transfer coefficients for the anodic and cathodic processes, respectively, of the rate-determining step, $n$ is the molar amount of electrons transferred per mole of reaction of the rate-determining step, and $i_{0}$ is the exchange current density linked to 9

$$
i_{0}=i_{0}^{\text {ref }}\left(\frac{c}{c_{\text {ref }}}\right)
$$

where $c$ is the concentration of dissolved $\mathrm{O}_{2}$ in the liquid close to the catalyst surface, $c_{\text {ref }}$ is the reference value of it, and $i_{0}^{\text {ref }}$ is the exchange current density evaluated at $c_{\text {ref }}$.

Similar to Lefebvre et al.'s transmission line model, ${ }^{2}$ if one is restricted to the discussion of a cathode where the electronic resistance is negligible, the matrix phase potential $\Phi_{1}$ does not change with position. If it is also assumed not to changed with time, a combination of Eq. 1 and 2 yields

$$
\frac{\partial^{2} \Phi_{2}}{\partial x^{2}}+\frac{\partial \ln \kappa}{\partial x} \frac{\partial \Phi_{2}}{\partial x}+\frac{a}{\kappa}\left(4 F j_{n}+C_{\mathrm{dl}} \frac{\partial\left(-\Phi_{2}\right)}{\partial t}\right)=0
$$

subject to boundary conditions

$$
\frac{\partial \Phi_{2}}{\partial x}=0 \quad \text { at } x=0
$$

and

$$
\frac{\partial \Phi_{2}}{\partial x}=-\frac{I}{\kappa} \quad \text { at } x=l
$$

where $I$ is the total current density applied to the cell and $l$ is the thickness of the active layer.

To make this model general, the second term in Eq. 6 provides the possibility that $\kappa$ is a function of $x$, i.e., the spatial position in the active layer. This is very likely to be true for the case where the Nafion ionomer solution is sprayed onto the active layer to enhance its ionic conduction. The amount of Nafion ionomer in the active layer is expected to vary with position after the desired total amount of Nafion is applied. Since $\kappa$ is the effective ionic conductivity in this model, both the porosity and tortuosity effects are already taken into account in this parameter.

It is convenient here to define $\kappa$ at any location of the active layer of a cathode as the product of an effective ionic conductivity $\kappa_{0}$, evaluated at the interfacial conditions between the active layer and membrane, and a distribution function $f(x / l)$, which is assumed to satisfy $0<f(x / l) \leqslant 1$. $\kappa$ is considered to have the largest value $\kappa_{0}$ in the active layer at $x=l$.

For the impedance study under OCP conditions, a sinusoidal perturbation of small amplitude, either in the form of current or potential, is applied to the cell. The response to the perturbation, which is linear due to the nature of a small perturbation, is measured to find the impedance response of the system. After substitution of each variable in Eq. 6-8 by a time-independent term, determined at OCP conditions, plus a time-dependent deviation term, we can cancel some time-independent terms in a way similar to a previous work. ${ }^{4}$ As a result, Eq. 6 yields

$$
\begin{aligned}
& \frac{\partial^{2} \widetilde{\Phi}_{2}}{\partial x^{2}}+\frac{\partial \ln f(x / l)}{\partial x} \frac{\partial \widetilde{\Phi}_{2}}{\partial x}-\frac{a}{R_{\mathrm{tc}} \kappa_{0}} \frac{1}{f(x / l)}\left(\tilde{\Phi}_{2}+R_{\mathrm{tc}} C_{\mathrm{dl}} \frac{\partial \widetilde{\Phi}_{2}}{\partial t}\right) \\
& =0
\end{aligned}
$$

where $\widetilde{\Phi}_{2}$ is the deviation of electrolyte phase potential from a value at OCP conditions, and $R_{\mathrm{tc}}$ is the charge-transfer resistance

$$
R_{\mathrm{tc}}=\frac{R T}{i_{0} n F}=\frac{R T}{i_{0}^{\mathrm{ref}} n F} \frac{c_{\mathrm{ref}}}{c_{0}}
$$

where $c_{0}$ is the concentration of dissolved $\mathrm{O}_{2}$ in the liquid electrolyte close to the catalyst surface at OCP conditions and is assumed to be uniform in this study. Equation 10 is obtained from the linear form of Eq. 4. (Responses are linear to the perturbation.)

Similarly, boundary conditions 7 and 8 yield

$$
\frac{\partial \tilde{\Phi}_{2}}{\partial x}=0 \quad \text { at } x=0
$$

and

$$
\frac{\partial \widetilde{\Phi}_{2}}{\partial x}=-\frac{\tilde{I}}{\kappa_{0}} \quad \text { at } x=l
$$

where $\tilde{I}$ is the deviation of current density from a value at OCP conditions. The impedance response of the porous cathode can be calculated by

$$
Z=\frac{\left.\widetilde{\Phi}_{1}\right|_{x=0}-\left.\widetilde{\Phi}_{2}\right|_{x=l}}{\widetilde{I}}=-\frac{\left.\widetilde{\Phi}_{2}\right|_{x=l}}{\widetilde{I}}
$$


where $\widetilde{\Phi}_{1}$, the deviation of matrix phase potential, is zero due to the assumption of a constant solid-phase potential.

A dimensionless analysis is carried out on Eq. 9, 11, and 12 to yield

$$
\frac{\partial^{2} \hat{\Phi}_{2}}{\partial y^{2}}+\frac{\partial \ln f(y)}{\partial y} \frac{\partial \hat{\Phi}_{2}}{\partial y}-\frac{l / \kappa_{0}}{R_{\mathrm{tc}} /(a l)} \frac{1}{f(y)}\left(\hat{\Phi}_{2}+R_{\mathrm{tc}} C_{\mathrm{dl}} \frac{\partial \hat{\Phi}_{2}}{\partial t}\right)=0
$$

$$
\frac{\partial \hat{\Phi}_{2}}{\partial y}=0 \quad \text { at } y=0
$$

and

$$
\frac{\partial \hat{\Phi}_{2}}{\partial y}=\hat{i} \quad \text { at } y=1
$$

where $\hat{i}$ is the dimensionless deviation current density and related to $\tilde{I}$ and its amplitude $\|\widetilde{I}\|$ by

$$
\hat{i}=\frac{\widetilde{I}}{\|\widetilde{I}\|}
$$

and where $y$ is the dimensionless coordinate and related to $x$ by

$$
y=\frac{x}{l}
$$

and where $\hat{\Phi}_{2}$ is the dimensionless deviation of the electrolyte phase potential and related to $\widetilde{\Phi}_{2}$ by

$$
\hat{\Phi}_{2}=\frac{\widetilde{\Phi}_{2}}{-\frac{l}{\kappa_{0}}\|\widetilde{I}\|}
$$

The dimensionless impedance response of a PEMFC cathode is

$$
\frac{Z}{l / \kappa_{0}}=\frac{\left.\hat{\Phi}_{2}\right|_{y=1}}{\hat{i}}
$$

where $Z$ is the actual dimensional impedance and normalized by $l / \kappa_{0}$ to yield a dimensionless impedance.

The conversion of Eq. 14-16 to the frequency domain yields

$$
\frac{\partial^{2} \hat{\Phi}_{2}}{\partial y^{2}}+\frac{\partial \ln f(y)}{\partial y} \frac{\partial \hat{\Phi}_{2}}{\partial y}-\frac{l / \kappa_{0}}{R_{\mathrm{tc}} /(a l)} \frac{1}{f(y)}\left(1+j R_{\mathrm{tc}} C_{\mathrm{dl}} \omega\right) \hat{\Phi}_{2}=0
$$

$$
\frac{\partial \hat{\Phi}_{2}}{\partial y}=0 \quad \text { at } y=0
$$

and

$$
\frac{\partial \widehat{\Phi}_{2}}{\partial y}=1 \quad \text { at } y=1
$$

where $\hat{\Phi}_{2}$ is a complex variable in the frequency domain and equal to the summation of a real part $\hat{\Phi}_{2, \mathrm{Re}}$ and an imaginary part $j \hat{\Phi}_{2, \mathrm{Im}}$, $j$ is $\sqrt{-1}$, and $\omega$ is the angular frequency.

We identify three characteristic groups for the cathode from Eq. 21 , a conductivity distribution function $f(y)$, a dimensionless angular frequency $\Omega=R_{\mathrm{tc}} C_{\mathrm{dl}} \omega$, and a ratio, to be represented by $R$, of an ionic resistance $l / \kappa_{0}$ to a charge-transfer resistance $R_{\mathrm{tc}} /(\mathrm{al})$. This ratio can be also interpreted as a dimensionless exchange current density according to Newman's notation. ${ }^{3}$ For clarity, Eq. 21 can be rewritten in a compact form

$$
\frac{\partial^{2} \hat{\Phi}_{2}}{\partial y^{2}}+\frac{\partial \ln f(y)}{\partial y} \frac{\partial \hat{\Phi}_{2}}{\partial y}-\frac{R}{f(y)}(1+j \Omega) \hat{\Phi}_{2}=0
$$

To find the dimensionless impedance response of the cathode, which is $\hat{\Phi}_{2, \operatorname{Re}}+j \hat{\Phi}_{2, \text { Im }}$ evaluated at $y=1$, one may collect the real components and imaginary components of Eq. 22-24 and let them be equal to zero separately. The real and imaginary parts of the actual dimensional impedance $Z$ of a cathode can be calculated by

$$
Z_{\mathrm{Re}}=\frac{l}{\mathrm{\kappa}_{0}} \hat{\Phi}_{2, \operatorname{Re}} \quad \text { and } Z_{\mathrm{Im}}=\frac{l}{\mathrm{\kappa}_{0}} \hat{\Phi}_{2, \mathrm{Im}} \quad \text { at } y=1
$$

For the case where $\kappa$ is uniform inside the active layer of the cathode, the second term in Eq. 24 vanishes and a simple analytical solution is available (see Appendix)

$$
\frac{Z}{l / \kappa_{0}}=\frac{\operatorname{coth}(\sqrt{R} \sqrt{1+j \Omega})}{\sqrt{R} \sqrt{1+j \Omega}}
$$

This also agrees with Eq. 13 of Springer's work. ${ }^{11}$

For the case where $\mathrm{\kappa}$ is nonuniform, we resort to a numerical solution. Three functions, $f(y)=y, f(y)=y^{2}$, and $f(y)=y^{4}$, are arbitrarily chosen to represent different nonuniformities of the ionic conductivity distribution inside the active layer. A numerical algebraic and differential equation solver in Fortran called GNES is used to solve our equations in each case, where a three-point finite difference is used to approximate each derivative variable. To speed up the numerical calculation, we took advantage of the banded structure of the Jacobian matrix. ${ }^{10}$

\section{Experimental}

Handmade V2-ELAT® gas diffusion electrodes with a Pt loading of $0.48 \mathrm{mg} / \mathrm{cm}^{2}$ (20\% Pt on carbon) from the E-TEK division of De Nora North America, Inc., were used as both the cathode and anode. Teflon is typically used in the active layer to manufacture an ELAT which helps form hydrophobic gas pores in the active layer (see Fig. 1). Different amounts of Nafion ionomer, ranging from 0.33 to 1.13 $\mathrm{mg} / \mathrm{cm}^{2}$ (dry weight), were sprayed onto the active layers of both the cathode and anode in our study. Similar to Lefebvre et al.'s model and experimental setup, ${ }^{2}$ the cathode is our working electrode and the anode fed with pure $\mathrm{H}_{2}$ is used as both a counter and a reference electrode. AC impedance and in situ cyclic voltammetry were conducted with a Gamry PC4/750 model DHC2 potentiostat. The area of all tested ELAT electrodes is $1.0 \mathrm{~cm}^{2}$. A porous current collector was used to allow both an even distribution of gas flow and good electronic contact with the electrodes.

Before the start of a test on a sample, the fuel cell was operated at $0.6 \mathrm{~V}$ on a $60 \mathrm{~A}$ fuel cell test station (Fuel Cell Technologies, Inc.) for $20 \mathrm{~h}$ to reach its steady-state operating current. The cell temperature was controlled at $70^{\circ} \mathrm{C}$ as well as the vapor saturation temperature for both the anode and cathode. The back pressures for the anode and cathode were set to 2.5 and $3.0 \mathrm{~atm}$, respectively. The air and $\mathrm{H}_{2}$ feedings were controlled at a flow rate (at standard temperature and pressure) of $220 \mathrm{~cm}^{3} / \mathrm{min}$.

Before the formal CV measurement was initiated, the air feeding on the cathode side was switched to $\mathrm{N}_{2}$ (vapor saturated), and then the fuel cell was cleaned by scanning for 100 cycles from -0.01 to $1.65 \mathrm{~V}\left(v s . \mathrm{H}_{2}\right.$ anode) at a scan rate of $100 \mathrm{mV} / \mathrm{s}$ once the operating current reduces to a negligible value. This step brings the Pt surface to a constant state. For comparison purposes, two scan rates, 50 and $100 \mathrm{mV} / \mathrm{s}$, were used in a formal CV measurement, where the cell potential was scanned from 0.04 to $0.55 \mathrm{~V}$ for 50 cycles. The back pressures for both the anode and cathode were set at zero in CV studies.

After the CV test, the back pressures of two electrodes were switched back to the cell's operating values, and the operating of the fuel cell at $0.6 \mathrm{~V}$ with air feeding was resumed for $30 \mathrm{~min}$ to allow the stabilization of the ionic conductivity profile. Then, the operation 


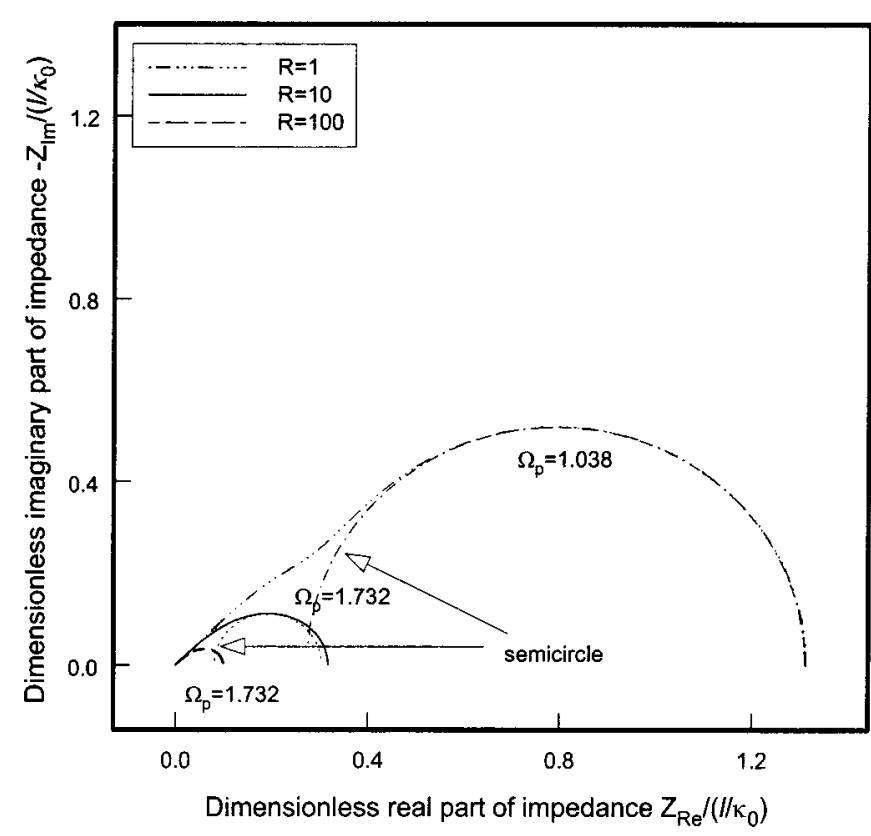

Figure 2. Dimensionless impedance simulation of a fuel cell cathode for a uniform ionic conductivity distribution $[f(y)=1]$ with the change of the ratio $R$ of an ionic resistance to charge-transfer resistance.

of the cell was interrupted by disconnecting the electronic load, and the cell was allowed to stabilize at $0.95 \mathrm{~V}$ (the current was zero) for $2 \mathrm{~min}$ before the impedance measurement was conducted. It was assumed that the ionic conductivity profile remained unchanged within a short time after interrupting the cell's operation. The perturbation amplitude used in the impedance measurement was set at 5 $\mathrm{mV}$ in a potentiostatic mode, and the frequency was scanned from 100,000 to $0.01 \mathrm{~Hz}$ with ten points per decade.

\section{Results and Discussion}

Model analysis.-Equations 22-25 indicate that the shape of actual impedance response is determined only by the parameter $R$ once the distribution function $f(y)$ is known. The simulated impedance response of a porous electrode with a uniform conductivity distribution $[f(y)=1]$ is presented in Fig. 2, where several cases with different $R$ values are compared. All the curves start from a $45^{\circ}$ line in the very high frequency region of the Nyquist plot. As the frequency decreases, all the curves become nonlinear and finally bend downward until the imaginary parts of impedance go to zero. When $R$ is very small, for example $R=1$, a semicircle is formed with the decrease of frequency after the $45^{\circ}$ line, and there is an obvious transition from the $45^{\circ}$ line to the semicircle region; when $R$ is large, for example $R=10$ or 100 , a highly depressed semicircle is formed after the $45^{\circ}$ line instead. The transition from the $45^{\circ}$ line to the depressed semicircle region does not exist. To demonstrate the nature of the impedance loop clearly, two semicircles with their centers located on the real axis and their heights equal to the maximum imaginary parts of the simulated impedance loops for the cases of $R=1$ and 10 are also drawn in Fig. 2. For comparison purpose, a characteristic dimensionless angular frequency $\Omega_{\mathrm{p}}$, at which the imaginary impedance reaches its maximum, was also indicated on each curve. It is known for a planar electrode that by using a simple equivalent circuit consisting of parallel $R_{\mathrm{tc}}$ and $C_{\mathrm{dl}}$ elements to consider both the charge-transfer and double-layer charging processes, we can model its impedance response by ${ }^{12}$

$$
Z=\frac{1}{1 / R_{\mathrm{tc}}+j C_{\mathrm{dl}} \omega}=\frac{R_{\mathrm{tc}}}{1+j \Omega}=\frac{1}{1+\Omega^{2}} R_{\mathrm{tc}}-j \frac{\Omega}{1+\Omega^{2}} R_{\mathrm{tc}}
$$

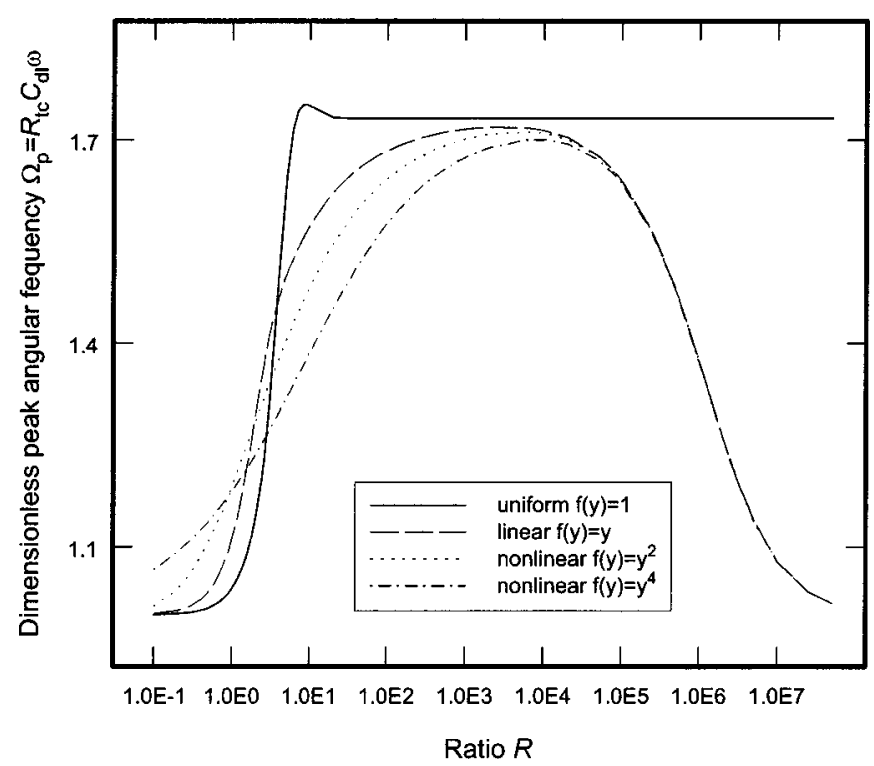

Figure 3. Dimensionless peak angular frequency with the change of the ratio $R$ of ionic resistance $\left(l / \kappa_{0}\right)$ to charge-transfer resistance $R_{\mathrm{tc}} /(a l)$.

that predicts an exact semicircle on a Nyquist plot. The $45^{\circ}$ line in the high frequency region, which is typical of a porous electrode, does not exist for a planar electrode. Moreover, $\Omega_{\mathrm{p}}$, the product of $R_{\mathrm{tc}}, C_{\mathrm{dl}}$, and $\omega_{\mathrm{p}}$, is always equal to unity for a planar electrode. This parameter provides us with an indication as to what extent the porous electrode deviates from a planar electrode. It is obvious from the $\Omega_{\mathrm{p}}$ values in Fig. 2 that a large deviation from a planar electrode behavior exists for the case such as $R=10$ and 100. Figure 2 also shows that the dimensionless impedance response $Z /\left(l / \kappa_{0}\right)$ decreases with the increase of $R$. This justifies our use of the $\mathrm{H}_{2}$ anode as the reference electrode if both the anode and cathode have similar $l / \kappa_{0}$ and $f(y)$. The $R$ value for the anode is much larger than the cathode due to its very small charge-transfer resistance. Because a larger $R$ value gives a smaller impedance response, the impedance response of a whole cell is predominately contributed from that of the cathode.

To understand the influence of the conductivity distribution profile on the porous electrode behavior, one may compare $\Omega_{\mathrm{p}}$ values among different cases. To find the numerical values of this parameter, we ran the impedance simulation of a porous electrode, assumed to have a fixed function $f(y)$, with the change of ratio $R$ and solved for $\Omega_{\mathrm{p}}$. The results are shown in Fig. 3. For a uniform $\kappa$ distribution in the active layer, there are two distinct regions, separated almost by a vertical line in between $R=2$ and $R=8$. When $R<1$, the ionic conduction is fast enough to allow the porous electrode to behave like a planar electrode. However, when $R$ $>20$, the maximum imaginary part of impedance occurs at an $\Omega_{\mathrm{p}}$ value $73.2 \%$ higher than that of a planar electrode. In a narrow range of $2<R<8$, there is an abrupt transition between the above two regions. For a nonuniform $\kappa$ distribution, i.e., a linear distribution $[f(y)=y]$, three regions are clearly identified and the transition from one to another is smoother than the case with a uniform distribution. When $R<0.1$, for $f(y)=y$, a planar electrode model is acceptable for treating the porous electrode. When $R>10^{7}$, the planar electrode model works again, due to the fact that the very low ionic conductivity, compared to relatively fast kinetics, makes the charge-transfer reaction and double-layer charging occur predominately at the interface between the electrode and membrane. When $0.1<R<10^{7}$, the value for $\Omega_{\mathrm{p}}$, first, rises with the increase of $R$, then reaches a plateau with a value around 1.7 , and finally decreases with the increase of $R$. The other two nonuniform distributions have 


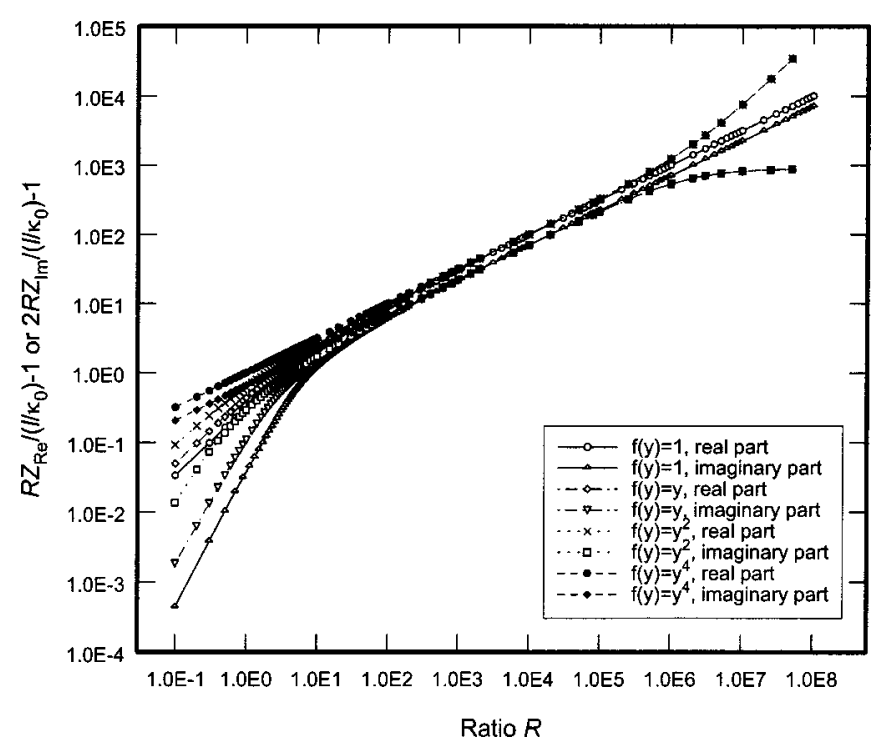

Figure 4. Comparison of deviations of the predictions of porous electrode impedance from those of planar electrode impedance. $\left(Z_{\mathrm{Re}}\right.$ is evaluated at $\Omega=0$ and $Z_{\mathrm{Im}}$ is evaluated at $\Omega=\Omega_{\mathrm{p}}$.)

similar behavior to the linear one except that the onsets of the middle region move to even lower $R$ values and the plateaus of the middle region becomes narrower.

Another way to look at the influence of ionic conductivity profile on the porous electrode behavior is shown in Fig. 4. It is known that the impedance response of a planar electrode on a Nyquist plot is an exact semicircle, which starts from the origin, with its center located on the horizontal axis. ${ }^{12}$ Therefore, the span of the whole impedance loop on the horizontal axis is simply the charge-transfer resistance, and the height of the curve is one half of that. The vertical axis in Fig. 4 displays a height or span difference, normalized by the height or span predicted from a planar electrode, between the porous electrode and the planar electrode. The horizontal axis displays the ratio $R$. In general, the porous electrode behavior is more and more unlike that of a planar electrode with the increase of $R$. For each conductivity distribution, the height of an impedance curve always deviates less from the prediction by a planar electrode model than the span of the real part. This can be explained by the contribution from ionic resistance, which is real, to the impedance span on the horizontal axis. When $R$ is very small (left lower section), different ionic conductivity distributions lead to different deviations of the porous electrode from planar electrode behavior, and the uniform and linear distributions cause smaller deviations compared to nonlinear ones. At intermediate large values of $R$, the deviation of the impedance span on the real axis is proportional to the deviation of the impedance height on the imaginary axis. To explain this, let us consider a situation where $\kappa_{0}, a$, and $l$ are held constant. In this case, the increase of $R$ value is only caused by the decrease of $R_{\mathrm{tc}}$. As a result of the decrease of the total charge-transfer resistance $R_{\mathrm{tc}} /(\mathrm{al})$, the actual observed resistance from the charge-transfer process also decreases. However, the decrease of the actual resistance from the charge-transfer process is slower than the increase of $R$ [or the decrease of $\left.R_{\mathrm{tc}} /(a l)\right]$, and every 100 times change of $R_{\mathrm{tc}} /(a l)$ causes only ten times the decrease of the observed charge-transfer resistance, according to the results for Fig. 4. This has a similar effect to reducing the active layer thickness with the increase of $R$ (more and more catalyst surface on the gas-backing side becomes not utilizable). As a result, both height and span deviations display a similar linear relation with the change of $R$. When we have a very large value of $R$ (right upper section), except for uniform ionic conductivity distribution, the deviation curves of the imaginary impedance go to a plateau, whereas those of the real impedance still increase

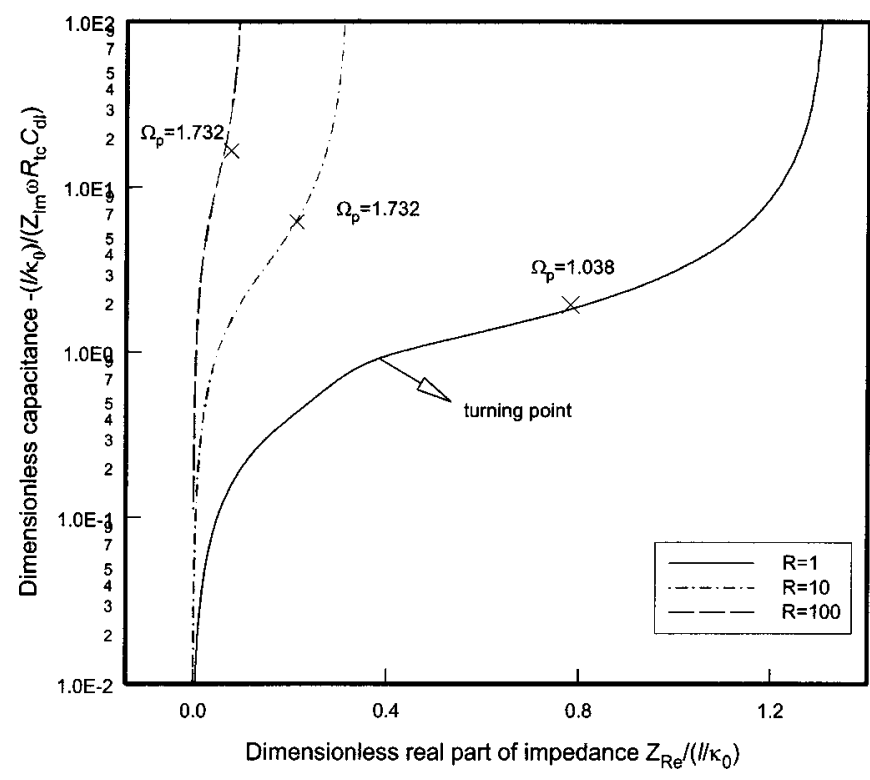

Figure 5. The dimensionless capacitance of the cathode with the change of dimensionless real part of impedance for a uniform ionic conductivity distribution $[f(y)=1]$.

with the increase of $R$ but following a nonlinear pattern. This is the region where the planar electrode theory works again. However, only the catalyst surface at the interface between the active layer and membrane is utilized.

Based on the model of a fuel cell cathode with an infinitly large value of charge-transfer resistance (nonpolarizable), Lefebvre et al. ${ }^{2}$ found that the observed capacitance of a porous electrode, interpreted from a $-1 /\left(Z_{\operatorname{Im}} \omega\right)$ vs. $Z_{\operatorname{Re}}$ plot, first increases with the increase of $Z_{\mathrm{Re}}$ (or the decrease of $\omega$ ) by following a linear profile, and then the profile becomes nonlinear until reaching a stop point [both $-1 /\left(Z_{\mathrm{Im}} \omega\right)$ and $Z_{\mathrm{Re}}$ do not change with $\left.\omega\right]$. The increase of observed capacitance with $Z_{\mathrm{Re}}$ can be explained by the gradual penetration of ionic conduction to the active layer with time. If the percentage contribution to the total double-layer capacitance from the catalyst Pt surface is assumed to be uniform in the active layer, the observed $-1 /\left(Z_{\mathrm{Im}} \omega\right)$ gives us an indication of how much catalyst surface is accessed. Our extended model of a fuel cell cathode predicts a linear region, a nonlinear region, and the appearance of a ramp, followed by the shooting up behavior of $-1 /\left(Z_{\operatorname{Im}} \omega\right)$ to infinity. These were demonstrated clearly for $R=1$ in Fig. 5, where other $R$ values for a uniformly distributed $\kappa$ profile were also considered. The vertical axis displays a dimensionless capacitance of the porous electrode, which has an ideal value of 1,10 , or 100 for the same value of $R$ in this order if the porous electrode behaves like an ideal planar electrode. This can be made clearer by rewriting the dimensionless group

$$
-\frac{l / \kappa_{0}}{Z_{\mathrm{Im}} \omega R_{\mathrm{tc}} C_{\mathrm{dl}}}=-\frac{1}{Z_{\mathrm{Im}} \omega} \frac{l / \kappa_{0}}{R_{\mathrm{tc}} /(a l)} \frac{1}{a l C_{\mathrm{dl}}}=-\frac{1}{Z_{\mathrm{Im}} \omega} \frac{R}{a l C_{\mathrm{dl}}}
$$

where $-1 /\left(Z_{\mathrm{Im}} \omega\right)$ is the capacitance in the actual dimensional form and is expected to cancel the term $a l C_{\mathrm{dl}}$ in the ideal case. To understand the appearance of a ramp as well as the shooting up behavior of $-1 /\left(Z_{\mathrm{Im}} \omega\right)$ with the increase of $Z_{\mathrm{Re}}$ as predicted by our extended model, it is helpful to start from considering the situation for a planar electrode. From Eq. 27, we can derive an equation for $-1 /\left(Z_{\operatorname{Im}} \omega\right)$

$$
-\frac{1}{Z_{\mathrm{Im}} \omega}=\frac{1+\Omega^{2}}{\Omega^{2}} C_{\mathrm{dl}}
$$




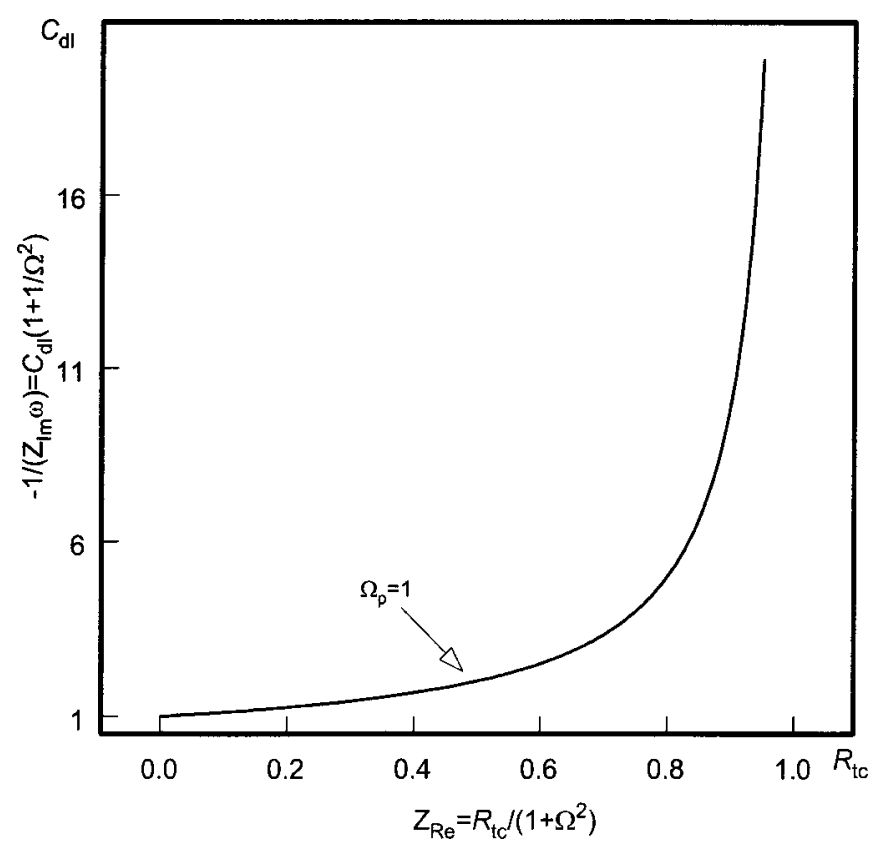

Figure 6. The capacitance $-1 /\left(Z_{\mathrm{Im}} \omega\right)$ for a planar electrode with the change of real part of impedance response $Z_{\mathrm{Re}}$.

The $-1 /\left(Z_{\mathrm{Im}} \omega\right)$ vs. $Z_{\mathrm{Re}}$ plot begins with a value $-1 /\left(Z_{\mathrm{Im}} \omega\right)=C_{\mathrm{dl}}$ in the very high frequency region, followed by a ramp with the decrease of frequency, and finally goes up to infinity at very low frequencies, which can be seen from Fig. 6. The gradual increase of $-1 /\left(Z_{\mathrm{Im}} \omega\right)$ with the increase of $Z_{\mathrm{Re}}$ (or the decrease of $\left.\omega\right)$ in the ramp region can be explained by the increasing branching of current to the charge-transfer reaction, and the shooting-up behavior can be explained by the ceasing of double-layer charging current at $\omega$ $=0$. A value of the double-layer capacitance larger than the true one is obtained from $-1 /\left(Z_{\mathrm{Im}} \omega\right)$ if there is branching of current to the charge-transfer reaction. [The use of $-1 /\left(Z_{\mathrm{Im}} \omega\right)$ to calculate the double-layer capacitance implies that the double layer charging is the only way for charge transfer.] All the $-1 /\left(Z_{\mathrm{Im}} \omega\right)$ vs. $Z_{\mathrm{Re}}$ plots predicted from our extended model are basically similar to Lefebvre et al.'s findings ${ }^{2}$ in the very high frequency region, where no current goes to the charge-transfer reaction, due to the existence of only the ionic conduction and double-layer charging processes. For the case where the ionic resistance is very small compared to the chargetransfer resistance, all the catalyst surface can be accessed by the double-layer charging before the onset of the charge-transfer reaction, and a separation by a turning point (immediately before the ramp) on a $-1 /\left(Z_{\mathrm{Im}} \omega\right) v s . Z_{\mathrm{Re}}$ curve is obvious between two regions with and without charge-transfer reaction current, as is shown for $R=1$ in Fig. 5. This provides us with a quantitative tool for the evaluation of the total catalyst surface area of a cathode. When the $R$ value becomes larger, the turning point is missing, which is the case for $R=10$ and 100 . Also, the determination of the total catalyst surface area by finding a turning point from a $-1 /\left(Z_{\mathrm{Im}} \omega\right) v s . Z_{\mathrm{Re}}$ plot fails because some of the catalyst surface near the gas-backing side are inaccessible even at $\omega=0$ due to slow ionic conduction compared with the relatively fast charge-transfer reaction.

If the dimensionless group on the vertical axis of Fig. 5 is divided by the ratio $R$, we have a plot of the ratio of observed capacitance to the total capacitance $v s$. the dimensionless real impedance. A plot with this interpretation is shown in Fig. 7 for four different ionic conductivity distributions. When $R=0.1$, a case where the ionic conduction is very fast compared to the charge-transfer reaction, uniform and linear $\kappa$ distributions yield an almost $100 \%$ accurate estimation of the total catalyst surface area $a$ by the method of

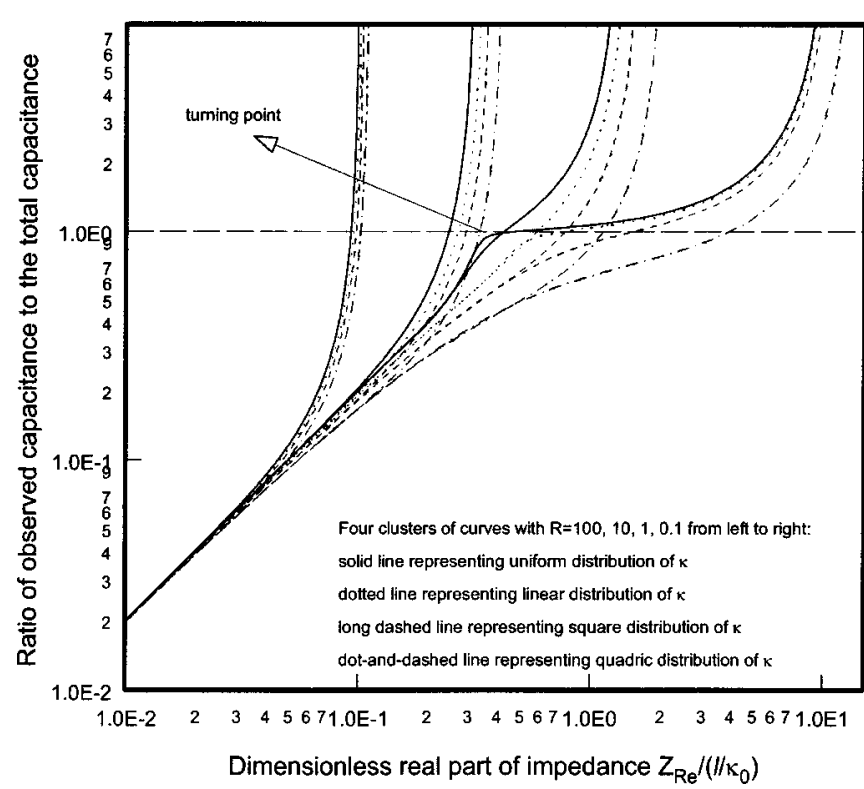

Figure 7. The ratio of observed capacitance $-1 /\left(Z_{\mathrm{Im}} \omega\right)$ to the total capacitance $a l C_{\mathrm{dl}}$ with the change of the dimensionless real part of impedance for different conductivity distribution profiles and different $R$ values.

finding the turning point, whereas an error in finding the total surface area is unavoidable for nonlinear $\kappa$ distributions due to the difficulty of locating the turning point. For $R=1$ under uniform $\kappa$ distribution, although one is still able to find an accurate estimation of the total catalyst surface area, there is some trouble in estimating correctly the other three distributions. Things are even poorer for the cases where the ionic resistance is very large compared to the charge-transfer resistance. Not all of the catalyst surface area is accessible for both $R=10$ and 100, regardless of $\kappa$ distributions. For example, it is impossible for us to measure the total catalyst surface area of a cathode from the impedance response at a large steadystate operating current. In this case, the overpotential driving force will make charge-transfer resistance very small, or consequently the $R$ value very big. We also observe from Fig. 7 that for a fixed value of $R$, the slope of each curve in the nonlinear region slightly decreases with the increase of nonuniformity of $\kappa$ distribution. This can be probably explained by a decreased value for $\kappa$ at any spatial coordinate with the increase of nonuniformity of its distribution. Similar to Lefebvre et al.'s findings, the increase of the slope of an actual dimensional curve $-1 /\left(Z_{\mathrm{Im}} \omega\right)$ vs. $Z_{\mathrm{Re}}$ in the high frequency linear region with the increase of $\kappa$ is also predicted by this extended model. This can be seen from Eq. 30, assuming $\mathrm{k}$ is the common slope of all the curves in the linear region of Fig. 7

$$
k=\frac{\frac{-1}{a l C_{\mathrm{dl}}} d\left(\frac{1}{Z_{\mathrm{Im}} \omega}\right)}{d\left(\frac{Z_{\mathrm{Re}}}{l / \kappa_{0}}\right)} \rightarrow \frac{-d\left(\frac{1}{Z_{\mathrm{Im}} \omega}\right)}{d Z_{\mathrm{Re}}}=k a C_{\mathrm{dl}} \kappa_{0}
$$

where $-d\left(1 / Z_{\mathrm{Im}} \omega\right) / d Z_{\mathrm{Re}}$ is the slope in an actual dimensional $-1 /\left(Z_{\mathrm{Im}} \omega\right)$ vs. $Z_{\mathrm{Re}}$ plot, and $\mathrm{k}$ is a constant, which is independent of the ionic conductivity distribution profile, $R$ value, and the active layer thickness $l$. Equation 30 reveals that the slope at any point in the high frequency linear region of the $-1 /\left(Z_{\mathrm{Im}} \omega\right) v s$. $Z_{\mathrm{Re}}$ plot is proportional to $\kappa_{0}$.

Experimental results.- $\mathrm{CV}$ was used to measure the active surface area of each cathode after operation in a fuel cell, and the typical voltammogram for an ELAT electrode is shown in Fig. 8. As one can observe, there are two pairs of hydrogen adsorption/ 


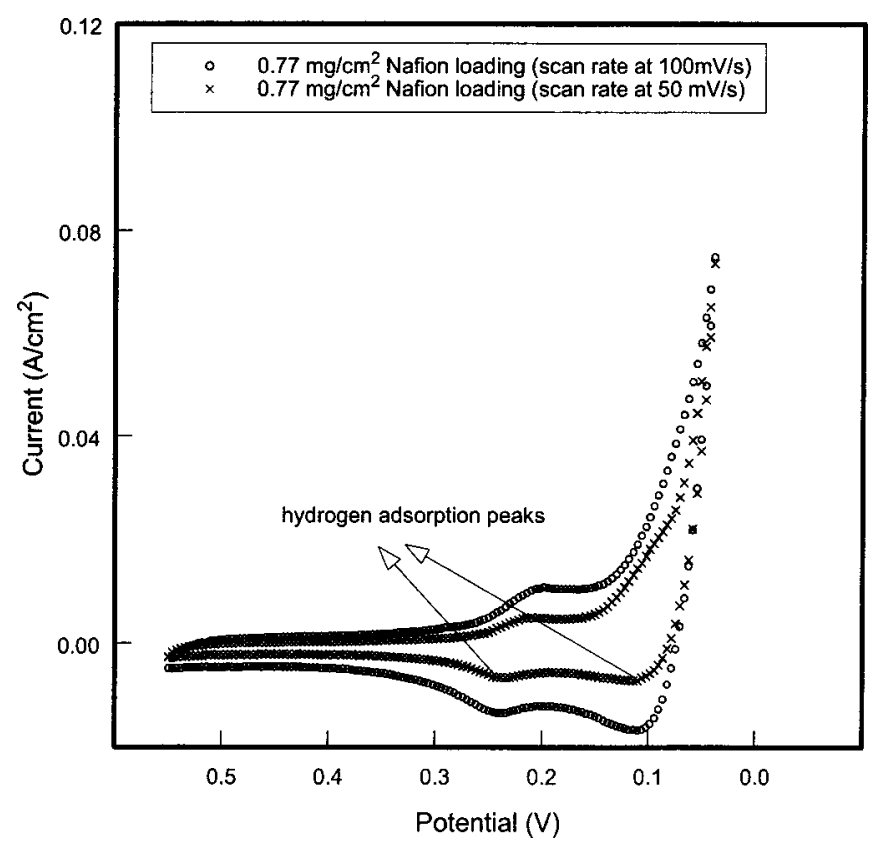

Figure 8. A demonstration of the cyclic voltammograms of a fuel cell with $\mathrm{N}_{2}$ fed to the cathode side and $\mathrm{H}_{2}$ fed to the anode side.

desorption peaks for each scan rate as is well known for platinum electrodes. To be consistent throughout our work, the hydrogen desorption peaks were used for the determination of surface area of the active catalyst sites. To find the surface area, a baseline is first constructed from the tangent to the left side plateau, and then one integrates the current difference between the baseline and two hydrogen desportion peaks (oxidation of adsorbed hydrogen) over time. With the assumption of monolayer adsorption of hydrogen, a value of 210 $\mu \mathrm{C} / \mathrm{cm}^{2}$ was used to convert the charge to the surface area. ${ }^{5}$ Two scan rates were applied for each test fuel cell to verify the reproducibility of the CV method. The surface area results are tabulated in Table I. In general, the surface area of an ELAT cathode measured by $\mathrm{CV}$ increases with the increase in Nafion content. This was expected because more of the catalyst surface can be accessed by protons with the increase of ionomer loading.

The Nyquist plots of the impedance response of a fuel cell at OCP are shown in Fig. 9, where several cases with different Nafion loadings are compared. Basically, each curve in Fig. 9 displays a semicircle-like profile, even if a slight deviation from an exact semicircle exists for each of them. To understand the nature of all the impedance loops in Fig. 9, we need to guess the $R$ values. If we assign a value, $0.106 \Omega \mathrm{cm}^{2}$, found by Springer et al. ${ }^{4}$ to $l / \kappa_{0}$ and assign the low frequency intercept on the real axis of Fig. 9 to $R_{\mathrm{tc}} /(a l)$, we find that the $R$ value is not larger than 0.01 for each case. Even if it is possible that we have even larger $l / \kappa_{0}$ values, it is unlikely for them to be larger than $10.6 \Omega \mathrm{cm}^{2}$, a value that still makes the $R$ value equal to 1 . Now it looks to us that the total

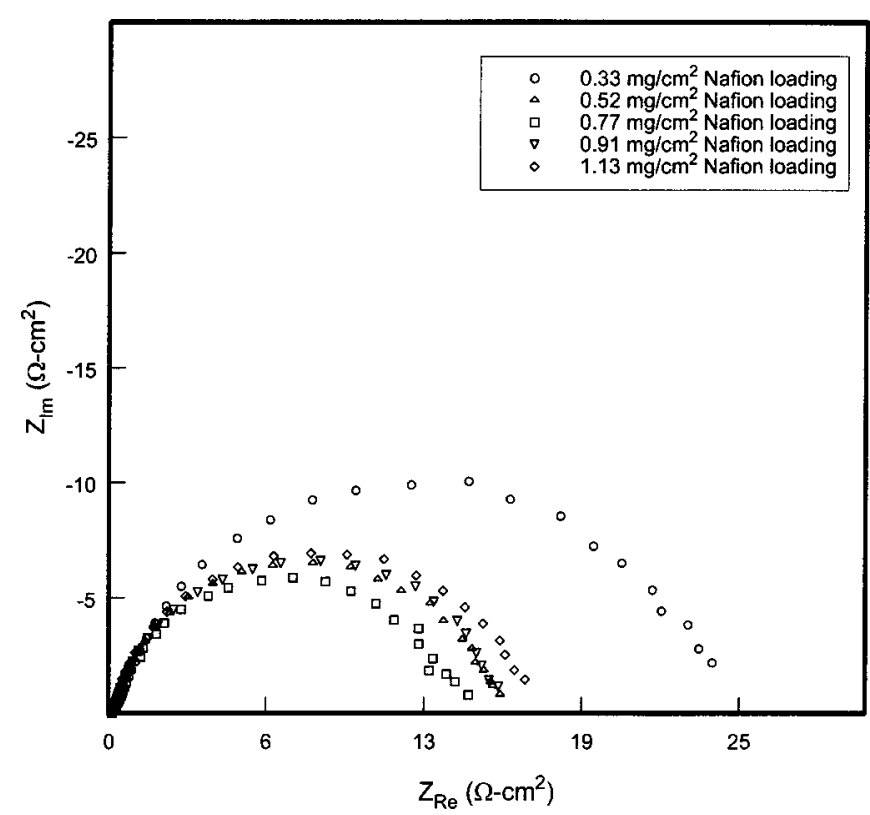

Figure 9. Comparison of Nyquist plots of a fuel cell with different Nafion loadings in the active layers of both the cathode and anode.

catalyst surface is accessible at OCP conditions and a planar electrode theory is valid here. We also observe from Fig. 9 that the fuel cell with $0.33 \mathrm{mg} / \mathrm{cm}^{2}$ ionomer loading shows a higher impedance than any of the other loadings, which indicates a smaller active surface area of $\mathrm{Pt}$ in the active layer. One might also notice that the increase of Nafion ionomer loading from 0.77 to $1.13 \mathrm{mg} / \mathrm{cm}^{2}$ does not necessarily lead to the decrease of the impedance of the cathode. We offer an explanation later.

The $-1 /\left(Z_{\mathrm{Im}} \omega\right) v s$. $Z_{\mathrm{Re}}$ plot to interpret the measured OCP impedance data is shown in Fig. 10. It is obvious that each curve displays a similar profile, where a linear region, a nonlinear region, a ramp, and a shooting-up behavior are identified. In the linear regions, the slope of each curve increases with the increase of ionomer loading, which agrees with our model prediction. The location of the ramp region for each of the high ionomer loadings, $0.77,0.91$, and $1.13 \mathrm{mg} / \mathrm{cm}^{2}$, is higher than either of the two low ionomer loadings, 0.33 and $0.52 \mathrm{mg} / \mathrm{cm}^{2}$. By extrapolating both the ramp region and linear region to a turning point, we can find the total double-layer capacitance of a cathode. These capacitance results are tabulated in Table I, which shows an increasing order of capacitance with the increase of ionomer loading and agrees with the trend observed from $\mathrm{CV}$ tests. Special care should be taken in order to compare these capacitance results with the surface area results measured from CV tests. The capacitance results tabulated in Table I are believed to reflect the ionic conductivity profile under the operating conditions of a cathode, since the impedance data were collected almost immediately after interrupting the operation of the cell. In contrast, deviation from the operating conditions might be reflected in the $\mathrm{CV}$

Table I. Comparison of the capacitance results calculated from impedance data with the surface area results calculated from CV measurement.

\begin{tabular}{|c|c|c|c|c|c|c|}
\hline & & \multicolumn{5}{|c|}{ Nafion ionomer loading $\left(\mathrm{mg} / \mathrm{cm}^{2}\right)$} \\
\hline & & 0.33 & 0.52 & 0.77 & 0.91 & 1.13 \\
\hline Capacitance & $-1 /\left(Z_{\mathrm{Im}} \omega\right)$ vs. $Z_{\mathrm{Re}}$ plot & 15.1 & 15.5 & 21.8 & 25.6 & 27.6 \\
\hline$\left(\mathrm{mF} / \mathrm{cm}^{2}\right)$ & $a l C_{\mathrm{dl}}=\operatorname{al}\left(R_{\mathrm{tc}} \omega\right)$ & 26.5 & 25.8 & 35.3 & 41.1 & 38.4 \\
\hline Surface area & $\mathrm{CV}$ at $50 \mathrm{mV} / \mathrm{s}$ & 57.4 & 61.9 & 76.2 & 109.5 & 119 \\
\hline$\left(\mathrm{cm}^{2} / \mathrm{cm}^{2}\right)$ & $\mathrm{CV}$ at $100 \mathrm{mV} / \mathrm{s}$ & 61.9 & 71.4 & 90.5 & 114.3 & 128.6 \\
\hline
\end{tabular}

Note: the surface area is evaluated as the total observed area per geometry area of the cathode. 


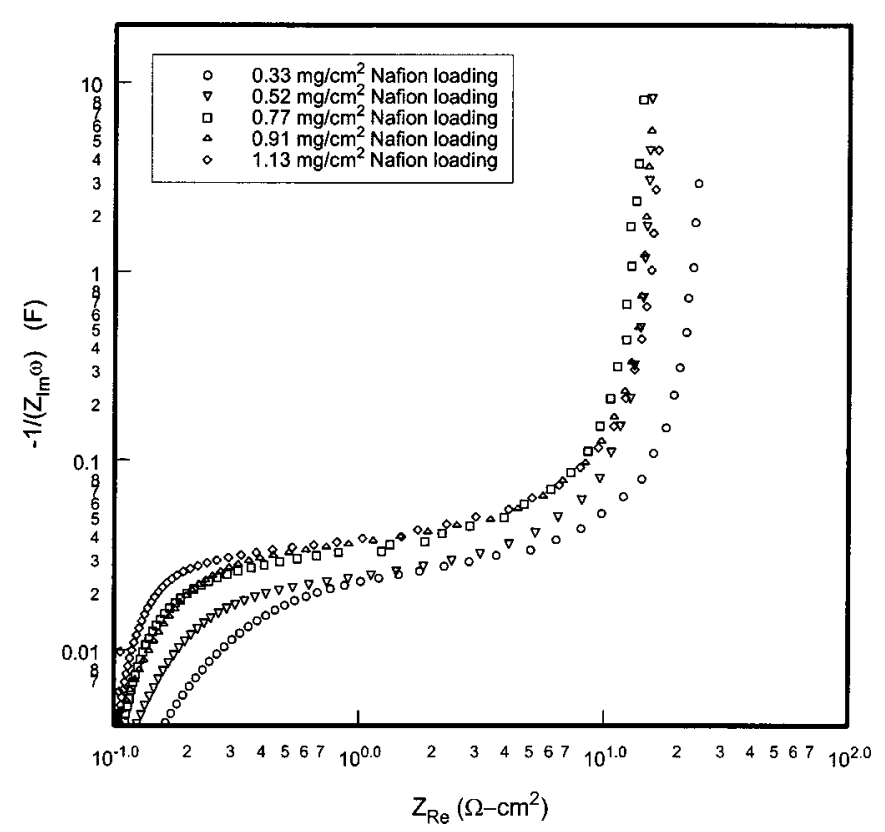

Figure 10. The $-1 /\left(Z_{\operatorname{Im}} \omega\right)$ vs. $Z_{\text {Re }}$ plots of a fuel cell with different Nafion loadings in the active layer of both the cathode and anode.

results, since the $\mathrm{CV}$ tests usually last a long period of time and especially since we need to cycle and condition the catalyst surface before any formal $\mathrm{CV}$ measurement.

If an impedance response has a semicircle-like shape, we can also find the total double-layer capacitance from the equation $a l C_{\mathrm{dl}}=\operatorname{all}\left(R_{\mathrm{tc}} \omega_{\mathrm{p}}\right)$ derived from a planar electrode theory, where $\omega_{\mathrm{p}}$ is the angular frequency at which the imaginary part of the impedance reaches its maximum value and $R_{\mathrm{tc}} /(a l)$ is the chargetransfer resistance read from the span of the impedance semicircle on the horizontal axis. The capacitance results calculated from this equation are also included in Table I for comparison purposes. As we can see from Table I, the capacitance results found from a $-1 /\left(Z_{\mathrm{Im}} \omega\right)$ vs. $Z_{\mathrm{Re}}$ plot are generally smaller than those calculated from the planar electrode theory, even if either set of capacitance results are generally able to predict the increase of capacitance with the increase of Nafion ionomer loading. The difference between two sets of capacitance results is probably due to an underestimation of $a l C_{\mathrm{dl}}$ by finding the turning point from a $-1 /\left(Z_{\mathrm{Im}} \omega\right) v s . Z_{\mathrm{Re}}$ plot. It is very likely that all the cathodes have nonlinear ionic conductivity distributions. The access of the total catalyst surface by double-layer charging is not yet completed before an appreciable current has branched to the charge-transfer reaction, and some more catalyst surface is still accessible in the ramp region with the decrease of $\omega$. To improve the accuracy of finding $a l C_{\mathrm{dl}}$ from a $-1 /\left(Z_{\mathrm{Im}} \omega\right) v s . Z_{\mathrm{Re}}$ plot, one might want to try an inert gas feeding to the cathode. In this case, all the catalyst surface is accessible since double-layer charging is the only way for charge transfer.

The ionic resistance $l / \kappa_{0}$ is a very important parameter. If we are able to find the value for it, a quantitative evaluation of the ionic conduction of a fuel cell cathode is possible. It might be possible to extract it from a simultaneous fit, together with other model parameters, from the steady-state impedance data in a way similar to that in Ref. 11. Unfortunately, the greater the number of parameters to be fitted in a model, the more difficult the convergence of the fitting. The OCP impedance data, measured almost immediately following the interruption of a cell's operation, simplifies our consideration from a complicated steady-state model, where many parameters play a role, to a simple model such as Eq. 24, where only a few parameters are important. It is even more favorable to us to have a situation with semicircle-like impedance spectra, since the time constant
$R_{\mathrm{tc}} C_{\mathrm{dl}}$ can be calculated approximately from the relationship $R_{\mathrm{tc}} C_{\mathrm{dl}} \omega_{\mathrm{p}}=1$ and the total charge-transfer resistance $R_{\mathrm{tc}} /(a l)$ can be read from the maximum imaginary part of impedance on the vertical axis of a Nyquist plot or the span of the semicircle on the horizontal axis. With such a situation, only the parameter $l / \kappa_{0}$ is left, and we can estimate it from the high frequency region, where the ramp in a $-1 /\left(Z_{\mathrm{Im}} \omega\right) v s$. $Z_{\mathrm{Re}}$ plot is not yet reached and the exact knowledge of $R_{\mathrm{tc}} /(\mathrm{al})$ is not required due to the relative importance of ionic conduction and double-layer charging. This is very important for us, because there is some difference between the $R_{\mathrm{tc}} /(a l)$ values found from the maximum imaginary part of impedance and from the span of a semicircle on the horizontal axis of a Nyquist plot. The method of nonlinear parameter estimation was used to extract the parameter $l / \kappa_{0}$ in this work by following a procedure similar to Ref. 10. To determine the accuracy of values obtained for this parameter, a $95 \%$ confidence interval ${ }^{10,13-16}$ for $\left(l / \kappa_{0}\right)$ was found by using

$$
\left(l / \kappa_{0}\right)=\left(l / \kappa_{0}\right)_{\mathrm{e}} \pm t_{1-0.05 / 2} S_{\mathrm{E}} \sqrt{a_{11}} \sqrt{\left(J^{\mathrm{T} J}\right)^{-1}}
$$

where $\left(l / \kappa_{0}\right)_{\mathrm{e}}$ is the point estimate of parameter $l / \kappa_{0}, t_{1-0.05 / 2}$ is the value of $t$ distribution for the determination of the $95 \%$ confidence interval that depends on the number of data points (see Eq. 7.73 on page 468 of Ref. 16), $S_{\mathrm{E}}$ is the standard deviation for the data set (see below), $J$ is the Jacobian matrix (see below), $J^{\mathrm{T}}$ is the transpose of $J,\left(J^{\mathrm{T}} J\right)^{-1}$ is the inverse of the product of $J^{\mathrm{T}}$ and $J$, and $a_{11}$ is a diagonal element of the matrix $\left(J^{\mathrm{T}} J\right)^{-1}$ (see p. 490 of Ref. 16). In this work, we used the model equation

$$
-1 /\left(Z_{\mathrm{Im}} \omega\right)=f\left(l / \kappa_{0}, \omega\right)
$$

where $-1 /\left(Z_{\mathrm{Im}} \omega\right)$ is the dependent variable, $\omega$ is the independent variable, and $f\left(l / \kappa_{0}, \omega\right)$ has an explicit form for a uniform ionic conductivity distribution and an implicit form for a nonuniform distribution. The Jacobian matrix $J$ was obtained by

$$
J_{\mathrm{i}}=\left\{\frac{\partial\left[-1 /\left(Z_{\mathrm{Im}} \omega\right)\right]}{\partial\left(l / \kappa_{0}\right)}\right\}_{\mathrm{i}}
$$

after evaluation of each impedance data point either analytically or numerically. ${ }^{10}$

The estimated results of $l / \kappa_{0}$ for the cathodes with three representative Nafion loadings are presented in Table II, where all four possible ionic conductivity distribution profiles are also considered. After the estimation of $l / \kappa_{0}$, the ratio $R$ was determined for each case, and the standard square error $S_{\mathrm{E}}^{2}$ was calculated from

$$
S_{\mathrm{E}}^{2}=\frac{1}{N-1} \sum_{i=1}^{N}\left[\left(\frac{-1}{Z_{\operatorname{Im}} \omega}\right)_{\exp }-\left(\frac{-1}{Z_{\operatorname{Im}} \omega}\right)_{\text {pred }}\right]^{2}
$$

where $\left(-1 / Z_{\operatorname{Im}} \omega\right)_{\exp }$ is the experimental value for the dependent variable, $\left(-1 / Z_{\mathrm{Im}} \omega\right)_{\text {pred }}$ is the predicted value for it, and $N$ is the number of data points. As is seen from Table II, the ratio $R$ values for all the three Nafion loadings and all the four ionic conductivity distributions are smaller than 0.1 . This consequently verifies the planar electrode behavior of our cathode at OCP conditions.

Since the impregnation of the ionomer in the active layer improves the hydrophilic property, we expect to observe an apparent decrease of $l / \kappa_{0}$ with the increase of the ionomer loading. Unfortunately, if we compare the values of $l / \kappa_{0}$ among the three ionomer loadings by assuming the same ionic conductivity distribution profile, this trend is not clear. To clarify this point, we have to realize that different ionomer loadings do not necessarily lead to the same ionic conductivity distribution profile. If we compare the square error $S_{\mathrm{E}}^{2}$ among all the four ionic conductivity distribution profiles for the same ionomer loading, we find that $f(y)=y^{2}$ has the least $S_{\mathrm{E}}^{2}$ for both $0.33 \mathrm{mg} / \mathrm{cm}^{2}$ and $0.77 \mathrm{mg} / \mathrm{cm}^{2}$ Nafion loadings, whereas 
$f(y)=y^{4}$ has the least square error for $1.13 \mathrm{mg} / \mathrm{cm}^{2}$ Nafion loading. If we use $S_{\mathrm{E}}^{2}$ as a criterion to discriminate one distribution over another, $f(y)=y^{2}$ is the true ionic conductivity distribution function for two lower Nafion loadings, 0.33 and $0.77 \mathrm{mg} / \mathrm{cm}^{2}$, and $f(y)=y^{4}$ is the true ionic conductivity distribution function for the highest Nafion loading, $1.13 \mathrm{mg} / \mathrm{cm}^{2}$. If we compare the $l / \kappa_{0}$ results among all the three cathodes with their ionic conductivity distribution having the least square error, the decrease of the parameter $l / \kappa_{0}$ with the increase of Nafion loading is now obvious. The high nonlinearity of ionic conductivity distribution $f(y)$ found here also justifies the explanation for an error of finding $a l C_{\mathrm{dl}}$ from a $-1 /\left(Z_{\mathrm{Im}} \omega\right)$ vs. $Z_{\text {Re }}$ plot.

Presumably a significant portion of Nafion ionomer sprayed on the cathode with a total loading of $1.13 \mathrm{mg} / \mathrm{cm}^{2}$ is located between the active layer and membrane. In this case, the volume fraction of gas pores near the interface is negligible compared to the other part of the active layer. As a consequence, the active layer near the membrane side may be very likely flooded with too much ionomer concentration. The dissolved $\mathrm{O}_{2}$ concentration on the surface of catalyst particles close to the membrane side will be unlikely to be at its saturation value before the impedance measurement due to the increased diffusion path caused by flooding. By assuming that the kinetics of a cathode at OCP conditions follows a Butler-Volmer equation, such as Eq. 4, the increase of charge-transfer resistance with the decrease of surface concentration of dissolved $\mathrm{O}_{2}$ is clearly shown in Eq. 10. This explains the results in Fig. 8, where the impedance of a cathode with $1.13 \mathrm{mg} / \mathrm{cm}^{2}$ Nafion loading is not smaller than a cathode with a lower loading such as $0.77 \mathrm{mg} / \mathrm{cm}^{2}$. This phenomenon also agrees with our observation made from steady-state impedance data. Therefore, it is not always good to increase the Nafion loading on a cathode, and an optimal amount of the Nafion ionomer to facilitate both the ionic conduction and $\mathrm{O}_{2}$ diffusion to the catalyst sites is necessary. A cathode with the optimal amount of Nafion ionomer should be able to display a semicircle-like impedance profile with minimum height on the imaginary axis as well as minimum full span on the real axis for both the OCP conditions and steady-state operating conditions. (Consider that the impedance is measured at the same potential.)

\section{Conclusions}

AC impedance spectroscopy measured almost immediately following the interruption of operating a PEMFC is potentially a convenient tool to evaluate the ionic conduction performance of a PEMFC cathode. Improvement of the accessibility of the total catalyst surface with the increase of Nafion ionomer loading was observed from both the capacitance results, interpreted from a $-1 /\left(Z_{\operatorname{Im}} \omega\right)$ vs. $Z_{\mathrm{Re}}$ plot, and the surface area results, measured from $\mathrm{CV}$ tests. By using the least square error as a criterion to discriminate one ionic conductivity distribution over another in a nonlinear parameter fit, the true distribution can be identified, which was used to explain our experimental observations.

\section{Acknowledgments}

The authors appreciate the convenience offered by the E-TEK Division, and the support by U.S. Department of Energy under contract no. DE-FC04-02AL67606.

University of South Carolina assisted in meeting the publication costs of this article.

\section{Appendix}

If a uniform ionic conductivity distribution $[f(y)=1]$ inside the active layer is present, Eq. 24 reduces to

$$
\frac{\partial^{2} \hat{\Phi}_{2}}{\partial y^{2}}=R(1+j \Omega) \hat{\Phi}_{2}
$$

subject to boundary conditions 22 and 23 .

From Eq. A-1, we can write the solution for $\hat{\Phi}_{2}$ as 


$$
\hat{\Phi}_{2}=c_{1} \sinh (\sqrt{R} \sqrt{1+j \Omega} y)+c_{2} \cosh (\sqrt{R} \sqrt{1+j \Omega} y)
$$

where $c_{1}$ is found to be zero by applying boundary condition 22 , and $c_{2}$ is determined by Eq. A-3 after applying boundary condition 23

$$
c_{2}=\frac{1}{\sqrt{R} \sqrt{1+j \Omega} \sinh (\sqrt{R} \sqrt{1+j \Omega})}
$$

With $c_{1}$ and $c_{2}$, Eq. A-2 is updated to

$$
\hat{\Phi}_{2}=\frac{\cosh (\sqrt{R} \sqrt{1+j \Omega} y)}{\sqrt{R} \sqrt{1+j \Omega} \sinh (\sqrt{R} \sqrt{1+j \Omega})}
$$

The dimensionless impedance response of the porous electrode is

$$
\left.\hat{\Phi}_{2}\right|_{y=1}=\frac{\operatorname{coth}(\sqrt{R} \sqrt{1+j \Omega})}{\sqrt{R} \sqrt{1+j \Omega}}
$$

The actual dimensional impedance response is

$$
Z=\left.\frac{l}{\kappa_{0}} \hat{\Phi}_{2}\right|_{y=1}=\frac{l}{\kappa_{0}} \frac{\operatorname{coth}(\sqrt{R} \sqrt{1+j \Omega})}{\sqrt{R} \sqrt{1+j \Omega}}
$$

which agrees with Eq. 26.

\section{List of Symbols}

a surface area of the catalyst Pt per unit volume of the active layer, $\mathrm{cm}^{2} / \mathrm{cm}^{3}$

$a_{11}$ a diagonal element of the matrix $\left(J^{\mathrm{T}} J\right)^{-1}$

$c$ concentration of the dissolved $\mathrm{O}_{2}$ in the liquid electrolyte near the catalyst surface, $\mathrm{mol} / \mathrm{cm}^{3}$

$c_{0}$ concentration of the dissolved $\mathrm{O}_{2}$ in the liquid electrolyte near the catalyst surface at OCP conditions, $\mathrm{mol} / \mathrm{cm}^{3}$

$c_{\text {ref }}$ reference concentration, $\mathrm{mol} / \mathrm{cm}^{3}$

$C_{\mathrm{dl}}$ double-layer capacitance of the active layer of a cathode per unit surface area of the catalyst Pt, F/cm²

$F \quad$ Faradaic constant, $96,487 \mathrm{C} / \mathrm{mol}$

$f(y)$ distribution function of the ionic conductivity, $0<f(y) \leqslant 1$

I current density applied to the cathode, $\mathrm{A} / \mathrm{cm}^{2}$

$\tilde{I}$ deviation current density of the cathode, $\mathrm{A} / \mathrm{cm}^{2}$

$\|\widetilde{I}\| \quad$ amplitude of the deviation current density, $\mathrm{A} / \mathrm{cm}^{2}$

$\hat{i}$ dimensionless deviation current density with unity amplitude

$i_{0}$ exchange current density of the rate-determining step, $\mathrm{A} / \mathrm{cm}^{2}$

$i_{0}^{\text {ref }} \quad$ exchange current density of the rate-determining step evaluated at a reference concentration $c_{\text {ref }}$ of the dissolved $\mathrm{O}_{2}$ close to the catalyst, $\mathrm{A} / \mathrm{cm}^{2}$

$i_{2}$ current density of the electrolyte phase, $\mathrm{A} / \mathrm{cm}^{2}$

$J$ Jabobian matrix

$j_{n} \quad$ wall flux of $\mathrm{O}_{2}$ on the catalyst Pt surface, $\mathrm{mol} / \mathrm{cm}^{2}$

$k$ a common slope of the curve in the high frequency region of Fig. 7

$l$ thickness of the active layer of the cathode, $\mathrm{cm}$

$n$ molar amount of electrons transferred per mole of reaction of the ratedetermining step

$N$ number of data points

$R$ ratio of an ionic resistance and charge-transfer resistance, $\left(l / \kappa_{0}\right) /\left(R_{\mathrm{tc}} / a l\right)$

$R_{\mathrm{tc}}$ charge-transfer resistance of the catalyst, $\Omega \mathrm{cm}^{2}$

$S_{\mathrm{E}}$ standard deviation of predicted value of total double-layer capacitance from the experimental one, $\mathrm{F} / \mathrm{cm}^{2}$

$t$ time, s

$t_{1-0.05 / 2}$ a value of $t$ distribution for the determination of a $95 \%$ confidence interval

$x$ spatial coordinate, $\mathrm{cm}$

$y$ dimensionless spatial coordinate
$Z$ impedance in complex form, $\Omega \mathrm{cm}^{2}$

$Z_{\mathrm{Re}}$ real part of the complex impedance $Z, \Omega \mathrm{cm}^{2}$

$Z_{\mathrm{Im}}$ imaginary part of the complex impedance $Z, \Omega \mathrm{cm}^{2}$

Superscripts

$\mathrm{T}$ transpose symbol

-1 inverse symbol

Subscripts

i $i$ th data point

Greek

$\alpha_{\mathrm{a}}$ transfer coefficient for anodic reaction

$\alpha_{c}$ transfer coefficient for cathodic reaction

$\kappa$ effective ionic resistance of the electrolyte, $\Omega^{-1} \mathrm{~cm}^{-1}$

$\kappa_{0}$ effective ionic resistance of the electrolyte evaluated at the active layer/ membrane interfacial conditions, $\Omega^{-1} \mathrm{~cm}^{-1}$

$\Phi_{1}$ matrix phase potential of the cathode, $\mathrm{V}$

$\widetilde{\Phi}_{1}$ deviation of the matrix phase potential of the cathode, $V$

$\Phi_{2}$ electrolyte phase potential of the active layer of a cathode measured with a reference $\mathrm{O}_{2}$ electrode placed locally, $\mathrm{V}$

$\widetilde{\Phi}_{2}$ deviation of the electrolyte phase potential of the active layer of a cathode, $\mathrm{V}$

$\hat{\Phi}_{2}$ dimensionless deviation of the electrolyte phase potential of the active layer of a cathode

$\hat{\Phi}_{2}$ complex variable for the deviation of the electrolyte phase potential of the active layer of a cathode

$\omega$ angular frequency, $\mathrm{rad} / \mathrm{s}$

$\omega_{\mathrm{p}}$ angular frequency with maximum value of imaginary part of impedance, rad/s

$\Omega$ dimensionless angular frequency

$\Omega_{\mathrm{p}}$ dimensionless angular frequency value at which the imaginary part of the impedance reaches its maximum

\section{References}

1. M. L. Perry and T. F. Fuller, J. Electrochem. Soc., 149, S59 (2002)

2. M. C. Lefebvre, R. B. Martin, and P. G. Pickup, Electrochem. Solid-State Lett., 2 , 259 (1999).

3. J. S. Newman, Electrochemical Systems, 2nd ed., p. 455, Prentice Hall, Englewood Cliffs, NJ (1991).

4. T. E. Springer, T. A. Zawodzinski, M. S. Wilson, and S. Gottesfeld, J. Electrochem Soc., 143, 587 (1996).

5. J. Shan and P. Pickup, Electrochim. Acta, 46, 119 (2000).

6. M. Watanabe, K. Makita, H. Usami, and S. Motoo, J. Electroanal. Chem., 197, 195 (1986).

7. M. C. Lefbvre, Z. Qi, and P. Pickup, J. Electrochem. Soc., 146, 2054 (1999)

8. M. Perry, J. Newman, and E. J. Cairns, J. Electrochem. Soc., 145, 5 (1998).

9. G. Murgia, L. Pisani, M. Valentini, and B. D'Aguanno, J. Electrochem. Soc., 149, A31 (2002).

10. Q. Guo, V. R. Subramanian, J. W. Weidner, and R. E. White, J. Electrochem. Soc. 149, A307 (2002)

11. T. E. Springer, in Tutorials in Electrochemical Engineering-Mathematical Modeling, R. F. Savinell, J. M. Fenton, A. C. West, S. L. Scanlon, and J. Weidner, Editors, PV 99-14, p. 208, The Electrochemical Society Proceedings Series, Pennington, NJ (1999).

12. A. J. Bard and L. R. Faulkner, Electrochemical Methods Fundamentals and Applications, John Wiley \& Sons, New York (1980).

13. T. I. Evans and R. E. White, J. Electrochem. Soc., 136, 2798 (1989).

14. M. C. Kimble, R. E. White, Y. M. Tsou, and R. N. Beaver, J. Electrochem. Soc. 137, $2510(1990)$

15. A. T. Haug and R. E. White, J. Electrochem. Soc., 147, 980 (2000).

16. A. Constantinides, Numerical Methods for Chemical Engineers with MATLAB Applications, Prentice Hall, Upper Saddle River, NJ (1999). 